\title{
Extension of the INCL4 model for spallation reactions up to $20 \mathrm{GeV}$
}

\author{
J. Cugnon and S. Pedoux ${ }^{\mathrm{a}}$ \\ Physics Department, University of Liège, Allée du 6 Août 17, Bât. B5, 4000 Liège 1, Belgium
}

\begin{abstract}
The Liège intranuclear cascade model has been improved by allowing for multipion production in nucleonnucleon collisions, a feature that is more and more relevant in nucleon-induced spallation reactions when the incident kinetic energy exceeds $2 \mathrm{GeV}$ or so. A phase space model is presented for multipion production and tested by comparing with experimental data.
\end{abstract}

\section{Introduction}

The Liège intranuclear cascade (INCL) model for spallation reactions, extensively described in ref. [1], has been developed primarily for applications to accelerator-driven systems. Therefore it is particularly suited to the incident energy range extending from $200 \mathrm{MeV}$ to $2 \mathrm{GeV}$, where it has been extensively tested by the HINDAS collaboration [2]. Possible other applications of INCL4 pertain to the description of the interaction of cosmic rays, either in the atmosphere or in space mission vehicles, since their average energy lies around $1 \mathrm{GeV}$ per nucleon. However, the cosmic ray spectrum is extending towards larger energy and it is generally considered that one has to treat the spectrum up to $10 \mathrm{GeV}$ at least to have reliable predictions [3]. To make INCL4 realistic up to $20 \mathrm{GeV}$, several improvements are in order: (i) introduction of multi-pion production in nucleon-nucleon collisions, (ii) treatment of resonances heavier than the Delta resonance, (iii) introduction of strangeness production channels. This improvement of INCL4 is also motivated by the possible investigation of when the subnucleonic degrees of freedom are starting to be relevant, a question which is still largely pending. In this contribution, we will report on our progress on the first point. A simple model for multipion production is described in section 2. Some results are shown and discussed in section 3. Finally, section 4 contains our conclusion.

\section{A model for multipion production}

\subsection{Pion production in INCL4}

Since the standard version of INCL4 focuses on applications below $2 \mathrm{GeV}$, the inelastic $\mathrm{NN}$ channel is restricted to one-pion production. Furthermore it is assumed that pion production proceeds through the production and the subsequent decay of a $\Delta$-resonance. This is largely legitimate, since the inelastic

${ }^{a}$ Presenting author, e-mail: spedoux@ulg.ac.be nucleon-nucleon channel is indeed largely dominated by the $\Delta$ production [5], except for the $\mathrm{T}=0 \mathrm{pn}$ system. Above, say $\sim 1.5 \mathrm{GeV}$, the pion production mechanism is not well known. Surely, heavier resonances are produced, which can decay by pion emission. However, the fraction of the inelastic cross section corresponding to the production of these resonances is badly known. Furthermore, most of these resonances are very broad (except for strange resonances). These considerations pushed us to investigate whether it is possible, if not reasonable, to describe the inelastic cross sections by direct multipion production, except of course for the part which is dominated by $\Delta$ product ion.

\subsection{The multipion production model}

To describe the inelastic NN cross sections in terms of multipion production, we need to determine the cross sections for creating $n$ pions as functions of the c.m. energy $\sqrt{s}$ and a model for generating $\mathrm{n}$ pions at a given energy. The first part of this program have largely been done by Bystricky et al. [6]. They identified the cross cross sections $\sigma_{T}(n, \sqrt{s})$ for creating n pions from NN collisions in isospin $T$ at c.m. energy $\sqrt{s}$, by summing known partial cross-sections with definite final states (such a $p p \rightarrow p p \pi^{+} \pi^{-} \pi^{0}$ ). Not all partial cross sections are known and they had to rely on isospin symmetry, as far as they could. We redid the analysis, using slightly more accurate data at low energy. This allowed us to avoid the determination of a slightly negative cross-section $\sigma_{0}(1, \sqrt{s})$ close to threshold. We have also used the following sum rules:

$$
\begin{aligned}
\sigma_{p p}^{\text {inel }}(\sqrt{s}) & =\sum_{n}^{n_{\max }} \sigma_{1}(n, \sqrt{s}), \\
\sigma_{p n}^{\text {inel }}(\sqrt{s}) & =\sum_{n}^{n_{\max }} \frac{1}{2}\left(\sigma_{0}(n, \sqrt{s})+\sigma_{1}(n, \sqrt{s})\right)
\end{aligned}
$$

where $n_{\max }$ is a function of the energy. The splitting of the inelastic $N N$ cross sections is given in figure 1 , for the $\mathrm{T}=1$ channel only. Similar results are obtained for the $\mathrm{T}=0$ channel. 


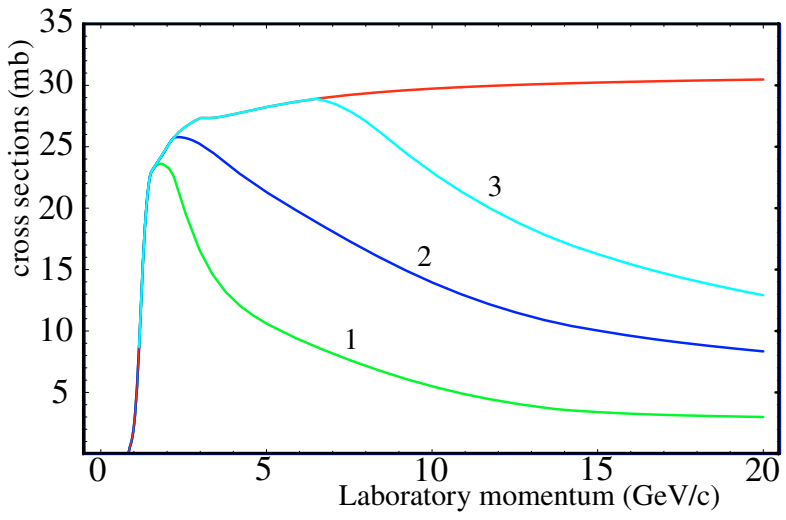

Fig. 1. Splitting of the total $\mathrm{T}=1$ nucleon-nucleon inelastic cross section (upper curve) in partial $\sigma_{1}(n, \sqrt{s})$ cross sections for multipion production. The successive lower curves correspond to the sums of the partial cross sections, up to $n=1,2$ and 3 , respectively.

For generating the final momenta of the particles in a $N N$ collision producing $n$ pions, we used a uniform phase space model, i.e., a probability for having 4-momenta $p_{i}$, $i=1,2, . . N, N=2+n$ proportional to:

$$
\begin{aligned}
& F\left(p_{1}, p_{2}, \cdots, p_{N}\right) \prod_{i=1}^{N} d^{4} p_{i}= \\
& \prod_{i=1}^{N} d^{4} p_{i} \delta\left(p_{i}^{2}-m_{i}^{2}\right) \theta_{+}\left(p_{i}^{2}\right) \delta^{4}\left(\sum_{j} p_{j}-P\right),
\end{aligned}
$$

with $P^{2}=s$ and $\theta_{+}$being the Heaviside function. The distribution $F$ is a relativistic invariant. It can thus be evaluated in the c.m. of $N N$ system. It is shown in ref. [7] that $F$ can then be rewritten as:

$$
\begin{aligned}
& F\left(p_{1}, p_{2}, \cdots, p_{N}\right) \prod_{i=1}^{N} d^{4} p_{i}= \\
& (2 \pi)^{N-1} p^{*}\left(M_{2}, m_{1}, m_{2}\right) p^{*}\left(M_{3}, M_{2}, m_{3}\right) \cdots p^{*}\left(\sqrt{s}, M_{N-1}, m_{N}\right) \\
& \times d M_{2} \cdots d M_{N-1} d \Omega_{N} d \Omega_{N-1} \cdots d \Omega_{2} .
\end{aligned}
$$

In this equation, $M_{i}$ is the invariant mass of the system composed of the first $i$ particles, $p^{*}\left(M, m, m^{\prime}\right)$ is the c.m. relative momentum of the particles $m$ and $m^{\prime}$ of total c.m. energy $M$ and $\Omega_{i}$ is the direction of the momentum of the $i$-th particle in the c.m. of the first $i$ particles. In order to generate the momenta of the particles, it is then sufficient to take the various directions at random and the invariant masses $M_{i}$ according to a weight given by the product of $p^{*}$ 's. In practice, we have adapted the KGENEV routine of the CERN library to the present case. Note that this procedure is applied for $n>1$ and for $\mathrm{n}=1$ in the $\mathrm{T}=0 \mathrm{NN}$ channel. For production of one pion in the $\mathrm{T}=1 \mathrm{NN}$ channel, we kept the usual scenario involving $\Delta$-production.

The determination of the charges of the pions is decoupled from the generation of their momenta. For $n=2$ or 3 , the probability for a final charge state can uniquely be determined by isospin conservation and the assumption that the weights are proportional to the Clebsch-Gordan coefficients for the

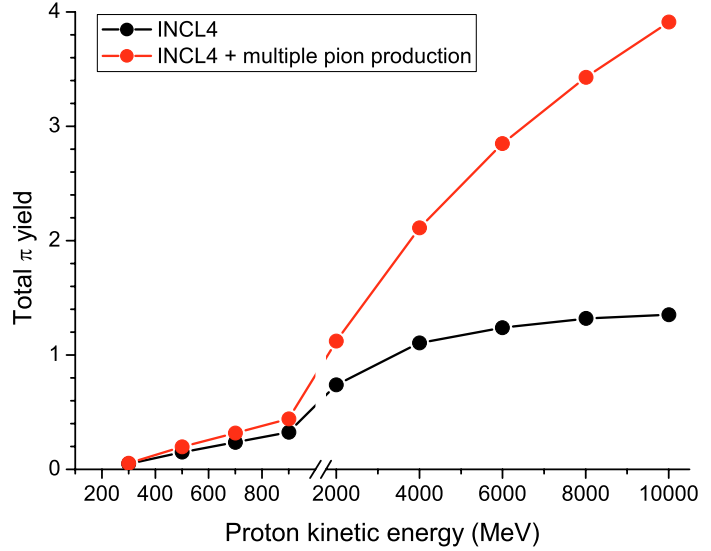

Fig. 2. Total pion multiplicity per reaction in $\mathrm{p}^{-209} \mathrm{Bi}$ collisions, as a function of the proton incident energy, evaluated with the standard INCL4 model [1] (black curve) and with our modified version (red curve). In this calculation, the multipion production has been restricted to $\mathrm{n}=2$ and 3 .

coupling of the isospin of the NN pair with the one of the pion system in the final state. For $\mathrm{n}$ larger than 3 , we used a simple statistical model.

\section{Results}

\subsection{Effects of the multipion production}

The basic effect of the introduction of multipion production is illustrated by figure 2, which shows the evaluated total pion multiplicity per reaction, with and without multipion production, as a function of the incident energy of protons bombarding ${ }^{209} \mathrm{Bi}$ nuclei. The pion multiplicity increases much faster in the modified case, for two reasons. First, the same nucleon-nucleon collision above $2 \mathrm{GeV}$ yields more pions in the modified version. Furthermore, in the same conditions, in the standard version, pions are produced with high kinetic energy and interact much less, since the $\pi-N$ cross section is underestimated over the $\Delta$ resonance region, in this version [1].

\subsection{Comparison with experimental data}

In order to validate our model, we have started to compare with existing data in the $1-10 \mathrm{GeV}$ range, since, below INCL4 gives reasonable values. A typical example is provided by figure 3, where we show the double differential cross section of negative pion production in proton-induced collisions on ${ }^{27} \mathrm{Al}$ nuclei at $4 \mathrm{GeV} / \mathrm{c}$. The agreement between our predictions and the experimental data is rather nice and encouraging.

These first results indicate that we have improved our INCL4 model in the pion sector above the GeV range. Of course, it is necessary to check whether we did not destroy our predictions for neutron and proton production. We have indeed calculated the double differential cross sections for proton production and compared with the measurements of 


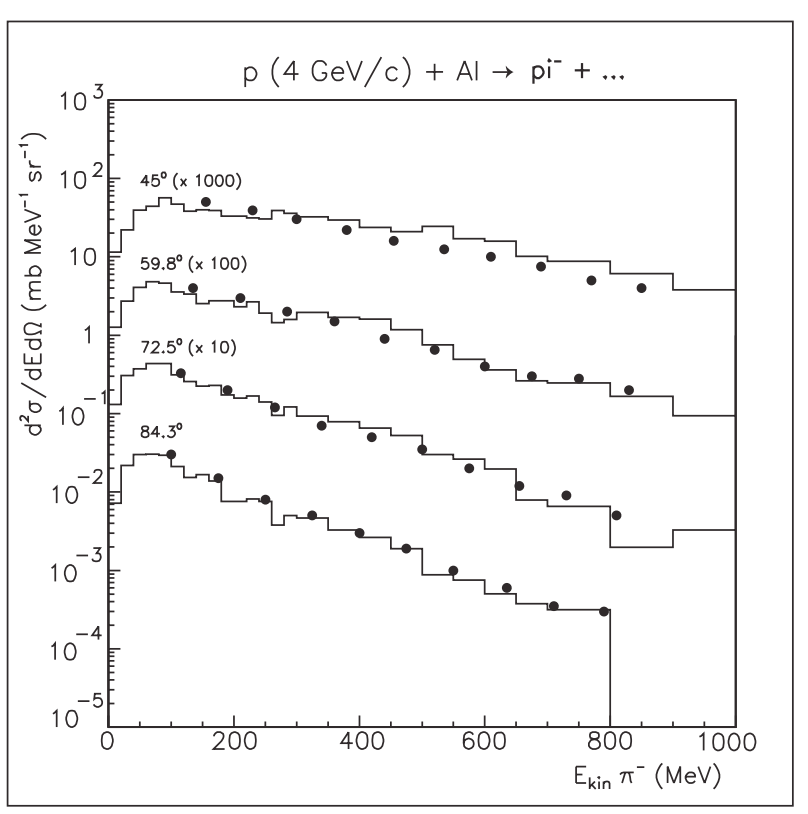

Fig. 3. Double differential cross sections for $\pi^{-}$production at four indicated angles in proton-induced reactions on ${ }^{27} \mathrm{Al}$ at $4 \mathrm{GeV} / \mathrm{c}$. Comparison between the experimental data of ref. [8] (heavy dots) and the predictions of our modified model (histograms).

Bayukov et al. [9] for proton collisions with $\mathrm{Pb}, \mathrm{U}$ and $\mathrm{C}$ nuclei, up to $7.5 \mathrm{GeV} / \mathrm{c}$. The results, not shown here, indicate that we reproduce satisfactorily the data, although the quality of the agreement is less than below $2 \mathrm{GeV}$ [1].

Another piece of comparison is provided by figure 4, where we compare the calculated excitation function for the negative pion yield in proton-induced collisions on Ta nuclei with experimental data. One can see that our results reproduce rather well the shape of the excitation function. The latter basically reflects the shape of excitation function of the elementary pion cross section in NN collisions, taking account of figure 2, and the fact that the inelastic NN cross section is basically constant above $1 \mathrm{GeV}$.

We also compared with the measurements of the HARP Collaboration at $13 \mathrm{GeV} / \mathrm{c}$, which are rather precise, but limited to very forward angles [11]. In this case, not shown here, we underpredict the production cross section by almost a factor 2. This result contrasts with those of figure 3 and indicate that we have probably to refine our phase space model.

\section{Discussion and conclusion}

We have presented here a first account of the improvement of the INCL4 model above $2 \mathrm{GeV}$, that we are currently pursuing. As we have said in the Introduction, the main missing features of INCL4 in this energy range are dealing with the NN inelastic channels: the absence of multipion pion production and the production of strangeness. We reported here on our progress on the first point.

Usually, multipion production is treated by allowing the production of resonances heavier than the $\Delta$-resonance, which

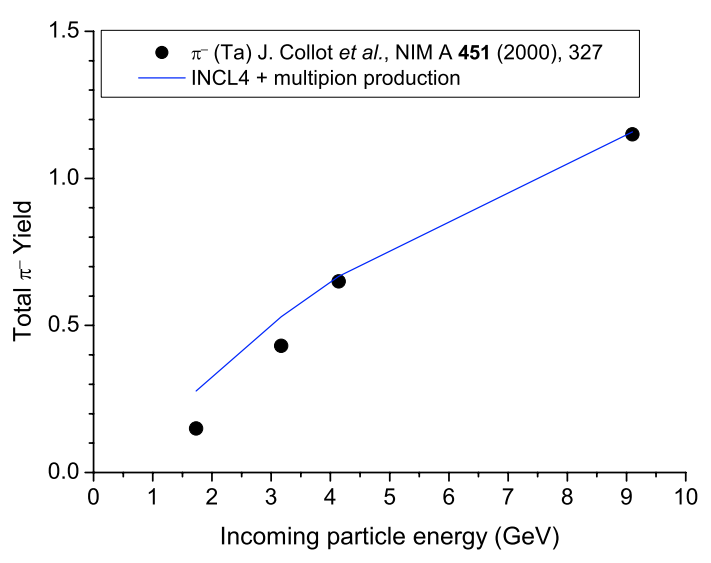

Fig. 4. Excitation function for the negative pion multiplicity in proton-induced collisions. Comparison between our predictions for Ta target (full line) and the experimental data (symbols), quoted in ref. [10].

may decay by emitting two or more pions. This seems to be the natural extension of what happens below $\sim 2 \mathrm{GeV}$. However, the cross sections for producing these heavier resonances are generally badly known, as are sometimes their widths and their branching ratio. Beyond introducing difficulties for the modelisation of the inelastic processes, this situation allows to raise the relevance of the latter in terms of resonances. On the contrary, several arguments are pointing toward a relatively minor role of these resonances. First of all, the width of these resonances is generally very large and increases with their masses. By the same token, their lifetime is very small, comparable if not smaller than the NN collision time. This leaves some doubt about the relevance of the explicit introduction of the heavier resonances in the proton-nucleus dynamics. In addition, there is no clear fin gerprint of the production of these heavier resonances in nucleon spectra, as it is for the $\Delta$-resonance.

In this work, we have decided to investigate the alternative scenario of the direct production of several pions in $\mathrm{NN}$ collisions. We have determined from the experimental data the phenomenological partial cross sections for producing $n$ pions, along the same lines as in ref. [6]. For the determination of the final states, except for the production of one pion in the $\mathrm{T}=1$ channel, we have adopted the simplest (and the extreme) model, namely the uniform phase space model. The distribution of charges in the final state is largely determined by isospin coupling coefficients (absolutely for $\mathrm{n}$ less or equal to 3 ).

We have tested our model by a preliminary comparison with data below $\sim 10 \mathrm{GeV}$. The fact that we obtained relatively good agreement with experimental data indicates that our model is reasonable. It should be realized however that, as far as the the pion multiplicity is concerned, there may be some ambiguity between our model and the usual model. In the former one, the pion multiplicity (in NN collisions) increases roughly linearly with the c.m. energy. In the latter model, the pion multiplicity increases with the mass of the heavy resonances, as underlined in ref. [12]. This yields approximately the same energy dependence of the pion multiplicity. However, the fact that we reasonably reproduce the angular dependance of the pion emission (see fig. 3) is probably 
in favor of our model, although this may presumably be obtained by some particular angular distribution of the heavy resonance production in the usual model. Finally, the fact that we underestimate the HARP data on pion production at very forward angles, at $13 \mathrm{GeV} / \mathrm{c}$, strongly indicates that our phase space model breaks down somehow around $10 \mathrm{GeV}$ or even at lower energy. This is perhaps not surprising, as it is expected that particle production is more and more forward (and backward) peaked as the energy increases.

In the next future, we want to correct our phase space model and investigate its validity up to $20 \mathrm{GeV}$. The following step will consist in including strangeness production. Although qualitatively important, this feature is still of minor importance below $20 \mathrm{GeV}$, as far as cross sections are concerned.

We want to thank Dr Y. Yariv for an interesting discussion. This work has been partially done in the frame of the EU IP EUROTRANS project (European Union Contract No. FI6W-CT-2004-516520). We acknowledge the EU financial support.

\section{References}

1. A. Boudard, J. Cugnon, S. Leray, C. Volant, Phys. Rev. C 66, 044615 (2002).

2. J.-P. Meulders et al., HINDAS Detailed Final Report, EU Report (2005).

3. T.K. Gaiser, Cosmic Rays and Particle Physics (Cambridge University Press, 1992).

4. J. Cugnon et al. (to appear in Advances in Space Research).

5. T. Ericson, W. Weise, Pions and nuclei (Clarendon press, Oxford, 1988), pp. 112-128.

6. J. Bystricky, P. La France, F. Lehar, F. Perrot, T. Siemarczuk, P. Winternitz, J. Phys. 48, 1901 (1987).

7. R. Hagedorn, Relativistic Kinematics (W.A. Benjamin, New York, 1963).

8. K. Niita et al., Phys. Rev. C 52, 2620 (1995).

9. Y.D. Bayukov et al., Sov. J. Nucl. Phys. 42, 116 (1985).

10. J. Collot, H.G. Kirk, N.V. Mokhov, Nucl. Instrum. Meth. A 451, 327 (2000).

11. The HARP experiment, http://harp.web.cern.ch/harp/.

12. J. Cugnon, J. Vandermeulen, Phys. Rev. C 39, 181 (1989). 